\title{
Decision Support for Watershed Management Using Evolutionary Algorithms
}

\author{
Misgana K. Muleta
}

and John W. Nicklow

\begin{abstract}
An integrative computational methodology is developed for the management of nonpoint source pollution from watersheds. The associated decision support system is based on an interface between evolutionary algorithms (EAs) and a comprehensive watershed simulation model, and is capable of identifying optimal or near-optimal land use patterns to satisfy objectives. Specifically, a genetic algorithm (GA) is linked with the U.S. Department of Agriculture's Soil and Water Assessment Tool (SWAT) for single objective evaluations, and a Strength Pareto Evolutionary Algorithm has been integrated with SWAT for multiobjective optimization. The model can be operated at a small spatial scale, such as a farm field, or on a larger watershed scale. A secondary model that also uses a GA is developed for calibration of the simulation model. Sensitivity analysis and parameterization are carried out in a preliminary step to identify model parameters that need to be calibrated. Application to a demonstration watershed located in Southern Illinois reveals the capability of the model in achieving its intended goals. However, the model is found to be computationally demanding as a direct consequence of repeated SWAT simulations during the search for favorable solutions. An artificial neural network (ANN) has been developed to mimic SWAT outputs and ultimately replace it during the search process. Replacement of SWAT by the ANN results in an $84 \%$ reduction in computational time required to identify final land use patterns. The ANN model is trained using a hybrid of evolutionary programming (EP) and the back propagation (BP) algorithms. The hybrid algorithm was found to be more effective and efficient than either EP or BP alone. Overall, this study demonstrates the powerful and multifaceted role that EAs and artificial intelligence techniques could play in solving the complex and realistic problems of environmental and water resources systems.
\end{abstract}

CE Database subject headings: Algorithms; Neural networks; Watershed management; Pollution control; Calibration; Computation.

\section{Introduction}

Agricultural source pollution, especially that associated with erosion and sedimentation, has been identified as a major component of nonpoint source (NPS) pollution in the United States (USEPA 2000). Erosion, in particular, is a complex phenomenon that is affected by many environmental factors including soil type, land use, topographic features, weather conditions, and human activities. A comprehensive approach for reducing erosion and sedimentation, therefore, should positively influence one or more of these governing factors, primarily those available for manipulation by humans. This study explores the potential role of optimal or near-optimal land use and management activity combinations in reducing erosion and sedimentation and their subsequent negative impacts. However, identification of preferred land use and management activities, their spatial (i.e., field-to-field) distribution across the watershed, and temporal (i.e., season-to-season) variation over the decision horizon is a daunting task. Such a procedure requires consideration of all the environmental, economic, and social implications of alternative scenarios. Furthermore, an evaluation of each possible decision scenario through experiment and monitoring programs is not feasible, leaving a modeling approach as the only reasonable means for NPS pollution control. The methodology used herein to solve the watershed management problem is based on the integration of a comprehensive watershed simulation model, an economic model, and a search mechanism (i.e., optimization method) that identifies the best alternative(s) among available possibilities, while giving due consideration to the social dynamics within the watershed.

Spatially distributed, long-term, continuous simulation models that have the capability to describe both the spatial and temporal variability of hydrologic variables are essential for the analysis of complex watershed systems. However, no matter how sophisticated they may be, models are simplifications of reality and users cannot expect their estimates to be accurate. Every model undergoes some kind of conceptualization or empiricism, and their results are only as good as model assumptions and algorithms, detail and quality of inputs, and parameter estimates. Calibration, which is basically a technique for bringing model estimates closer to the actual behavior of the study area by manipulating model parameters (Refsgaard 1997), is therefore an inevitable necessity. Calibration of distributed models is a complicated procedure since the number of uncertain parameters that need to be calibrated is large. The Soil and Water Assessment Tool (SWAT) (Arnold et al. 1998), a watershed simulation model developed by 
the U.S. Department of Agriculture (USDA) and the model used in this study, is a typical example of a spatially distributed model. In order to avoid limitations of existing manual (i.e., trial and error) calibration methods, an automated technique that uses a genetic algorithm (GA) (Holland 1975; Goldberg 1989) is developed herein for the calibration of daily flow volume and daily sediment yield estimates of SWAT. In addition, application of parameter reduction techniques, including parameterization and sensitivity analysis, as part of the technique effectively reduces the number of parameters to calibrate.

Used alone in their traditional capacity, calibrated simulation models are inefficient and can be ineffective for identifying a best set of alternatives. In complex water resources management systems, in which there may be a large number of potential management alternatives, determination of an optimal or near-optimal solution requires a more systematic decision-making framework such as the integration of a powerful optimization method with the simulation model. Traditional optimization methods - such as exhaustive search, iterative search, and gradient based techniques, and nonguided random search methods - are generally unsatisfactory for solving large, nonlinear, and nonconvex realistic problems. In contrast, evolutionary algorithms (EAs), search mechanisms that apply the principle of natural selection to improve system performance, are believed to work better in the situations where traditional methods have difficulty (Schwefel 2000). For the watershed management problem, for example, it is difficult, if not impossible, to derive a well-behaved (i.e., convex and unimodal) function that explains a required output variable (e.g., sediment yield) as a result of the governing system dynamics. Therefore, EAs tend to be an ideal choice for solving the problem presented in this study.

Consideration of the socioeconomic implications of watershed planning and management activities in multiple-owner largely private watersheds is quite challenging since different stakeholders may have varying priorities. For example, farmers may be more concerned with profit they generate from their farms, while the more environmentally conscious may be more inclined towards preserving environmental integrity of the watershed. To be successful, planning and management processes under such conflicting objectives require an approach that merges economic, social, and environmental priorities into a single framework that is relevant for farm-level, as well as watershed-level, analysis and decision making. Furthermore, it may be essential to evaluate alternatives from many perspectives, including single and multiple criteria, or on a field-by-field or watershed-scale basis.

Convinced by this philosophy, the writers have previously developed single objective (Nicklow and Muleta 2001) and multiobjective (Muleta and Nicklow 2002a) computational models for the control of erosion and sedimentation from watersheds. The models were designed as a guide for identifying best management practices to be implemented in farm fields so as to reduce anthropogenic-induced erosion and sediment yield without sacrificing landowners' economic benefits. The single objective model was a result of integrating SWAT with a GA, whereas the multiobjective model was developed by interfacing SWAT with a powerful multiobjective search technique known as Strength Pareto Evolutionary Algorithm (SPEA) (Zitzler and Thiele 1999). Both models were based on a farm-field scale decision-making framework and the resulting analyses were based on a noncalibrated SWAT model.

In this paper, the writers bring together various components of previous work into an overall decision support system and methodology. Specifically, this paper extends the integrative computa- tional methodology to the watershed level rather than farm-level analysis, thus making the decision-support system more comprehensive. The types of objectives considered at the watershed scale are the identification of farm fields in the watershed: (1) Where conservation programs should be focused from a pollution reduction perspective: (2) that are agriculturally more productive, and (3) where implementation of conservation programs may be the most cost effective (i.e., more reduction of sedimentation could be achieved with little loss of agricultural profit).

Assumptions and farm management practices used in previous models have also been revised based on interviews conducted with local Natural Resources Conservation Service (NRCS) personnel and other farm officers, thus improving the practicality of the decision support system. In addition, the applications presented here are based on a calibrated SWAT model, a task that was accomplished using an automatic calibration technique that relies on a GA (Muleta and Nicklow 2002b). Finally, application of the methodology generally reveals the success of the decision support system in addressing their corresponding objectives. However, the models are found to be computationally demanding, thus threatening their practical utility. The computational demand is mainly due to required iterative execution of the watershed simulation model, SWAT, as part of the search for preferred land use and management solutions. In order to resolve computational time concerns, a previously developed intermediate model $(\mathrm{Mu}-$ leta and Nicklow 2002c) that is based on an artificial neural network (ANN) is extended to embrace the revisions and the calibrated model. The ANN-based model is used to replace SWAT and mimic its computations in a fraction of the time required by the USDA model. For the ANN, a novel training algorithm is developed that is a result of hybridizing evolutionary programming (EP) (Fogel 1994) and a gradient-based training algorithm known as the back propagation (BP) (Rumelhart et al. 1986).

\section{Demonstration Watershed and Data Description}

Big Creek watershed, a 133- $\mathrm{km}^{2}$ basin located in Southern Illinois, is used for the demonstration of the decision support system developed in this study. This watershed not only contributes significant amounts of flow to the Lower Cache River, but also carries a higher sediment load than other tributaries in the area. According to data from 1985 to 1988 , Big Creek watershed contributed more than $70 \%$ of sediment inflows into the Lower Cache (Demissie et al. 2001). Large quantities of this sediment are deposited in aquatic and wetland habitats found in the Lower Cache River, threatening to eliminate the high-quality natural communities that inspired the designation of this area as a State Natural Area and Land and Water Reserve, a National Natural Landmark, an Important Bird Area, and a Wetland of International Importance (Guetersloh 2001). The watershed is characterized as an agricultural basin since the percentage of urban land use is insignificant. In addition, because of its high sediment yield and significant influence on the Lower Cache River, multiple state agencies and planning organizations have identified the Big Creek as a priority area for improved watershed management. It is now undergoing extensive study as part of the Illinois Pilot Watershed Program, through cooperation among the Illinois Department of Natural Resources (IDNR), the Illinois Department of Agriculture, Illinois Environmental Protection Agency (ILEPA), and the NRCS (IDNR 1998).

Application of SWAT to a watershed requires topographic, soil, land use, and climate data for the basin. In addition, stream 
flow and sediment concentration data are required for calibration efforts. For the Big Creek watershed, a 10-m-resolution Digital Elevation Model from NRCS, 30-m-pixel land use maps for the years 1999 and 2000 from National Agricultural Statistics Service, and a 30-m-resolution soil map from NRCS were obtained. Daily historical data related to precipitation, maximum temperature, minimum temperature, wind speed, humidity, and solar radiation were obtained from the Midwest Climate Center for nearby climate stations. Finally, daily flow volume and daily sediment concentration for water years 1999, 2000, and 2001 were obtained from the Illinois State Water Survey (ISWS) for a gauging station that drains approximately $65 \%$ of the watershed.

\section{Simulation Model Calibration}

SWAT is a continuous-time spatially distributed simulator developed to assist water resource managers in predicting the impacts of land management practices on water, sediment, and agricultural chemical yields (Arnold et al. 1998). SWAT makes use of watershed information, such as weather, soil, topography, vegetation, and land management practices, to simulate a variety of watershed processes including surface and subsurface flow; erosion and sedimentation of overland, as well as channel, flows; crop growth for user specified agricultural management practices; and nutrient cycling for various species of nitrogen and phosphorus, among others. Spatially, the model divides a watershed into subwatersheds, or subbasins, based on topographic information. The subwatersheds could be further classified into smaller spatial modeling units known as Hydrologic Response Units (HRUs) depending on the heterogeneity of land uses and soil types within the subbasins. At the scale of an HRU, watershed variables - such as soil types and properties, land use - and related management features, weather, and topographic parameters are considered homogeneous. For additional details regarding SWAT, the reader is referred to Arnold et al. (1998).

\section{Parameter Reduction Mechanisms}

Effective calibration of distributed models like SWAT begins by developing a proper mechanism for reducing the number of parameters to be calibrated. Screening which model parameters to estimate based on field data alone and which to determine based on calibration is the first logical step in that direction. In this study, a detailed investigation of SWAT's documentation has assisted in the identification of parameters that can be estimated with confidence based on available data alone. As a result, 42 parameters whose estimation from readily available data alone may pose significant uncertainty have been identified. 15 of these 42 parameters assume uniform values over the watershed, while values of the remaining 27 parameters differ from subbasin to subbasin and depend on soil properties and land use. Using the Geographic Information System interface of SWAT, the Big Creek watershed was divided into 78 subbasins, with each subbasin representing one HRU. Classification of the watershed into these different modeling units implies that each of the 27 parameters may assume different values for the 78 subbasins of the watershed. The problem is made even more complex since soil properties not only vary from soil type to soil type, but also from layer to layer of the same soil.

As a second step in reducing the number of spatially varying variables to calibrate, parameterization has been accomplished by using the concept of a representative HRU. In parameterization, a representative hydrologic unit is selected, upon which the model assumes homogeneity of parameters and variables. A relationship between parameters of this representative modeling unit and other homogeneous units in the watershed is developed using available information about the parameters. As an example, the curve number $(C N)$ and Manning's roughness coefficient $(n)$ of the representative HRU and other HRUs can be developed based on $C N$ and $n$ values recommended in the literature for conditions of the corresponding HRUs. Relationships for soil properties of the representative soil to other soils and from a representative layer of a given soil to other layers of the same soil are derived using the soil database that is supplied with the SWAT model. In this way, once parameter values for the representative HRU are determined, values for the remaining HRUs can be obtained from the relationship. Alternatively stated, only the 27 parameters of the representative HRU are involved in the calibration procedure. Yet, it may still be difficult to conduct calibration using all the 27 representative values, as well as the remaining 15 uniform watershedscale parameters. Particularly for watersheds that lack long periods of recorded data, which is the case for the Big Creek basin, it is essential to reduce the number of parameters to calibrate as much as possible. Therefore, further reduction of parameters through sensitivity analysis is conducted.

For sensitivity analysis, stepwise regression (Helton and Davis 2000) has been implemented. Maximum and minimum values for the 42 parameters were assigned based on values recommended in the literature and prior knowledge of the watershed. All of the parameters were assumed to follow a uniform distribution. From the distribution and the ranges assigned for the parameters, Latin hypercube sampling was applied to generate 300 input samples. For each of these input samples, the SWAT model was executed to provide an output to be used during the sensitivity analysis and which also serves as a fitness, or performance, measure to be used during calibration. Here, fitness is expressed as the sum of absolute deviations (i.e., residuals) between corresponding values of model estimates and measured responses for both sediment yield and flow volume. Based on the conception that rank-based regression analysis is superior when the input-output relationship is nonlinear (Iman and Conover 1979), ranks of the input-output pairs were used during the subsequent stages of the sensitivity analysis rather than working with actual values.

For stopping criteria of this analysis, flow volume was found to be significantly influenced by only 9 of the 42 parameters. Since sediment yield heavily depends on daily flow volume, there is little justification for calibrating both watershed responses for the same parameters. As a result, the parameters chosen for fitting flow data were not involved in the sensitivity analysis conducted for sediment yield, and only the remaining 33 parameters were analyzed for sediment yield. Six parameters were found to be significant for sediment yield.

\section{Calibration Procedure and Results}

Parameter estimation follows the determination of which model parameters to calibrate. Parameter estimation can be conducted either manually or in an automatic fashion. In manual calibration, essential model parameters would be adjusted by trial-and-error methods until model simulations satisfactorily match the measured data. This is by far the most widely used calibration approach for complex models (Refsgaard and Knudsen 1996; Refsgaard 1997; Senarath et al. 2000; Santhi, et al. 2001). Manual calibration, however, is a time consuming and very subjective procedure, and its success highly depends on the experience of 


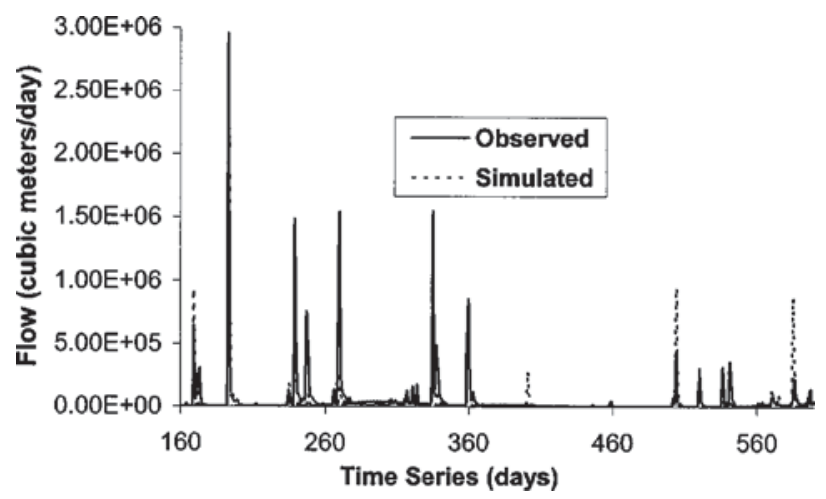

Fig. 1. Comparison of calibrated and measured values for daily streamflow

the modeler and their knowledge of the study watershed, model assumptions, and algorithms used. Automatic calibration, in contrast, involves the use of a search algorithm to explore the numerous combinations of parameter levels in order to achieve the set of which is best in terms satisfying the criterion of accuracy. Automatic calibration offers many advantages over the manual approach. It is computationally fast, it is less subjective, it does not require a highly experienced modeler, and since it makes an extensive search of the existing possibilities, it is highly likely that results will be better than those which could be manually obtained. Use of proper search criterion (e.g., objective function), use of a search technique that makes a global search (e.g., GA) high-quality data, and assignment of physically realistic ranges of parameter values are crucial for successful implementation of automatic calibration.

For this study, an automatic calibration model that uses a real coded GA is developed for daily streamflow and daily sediment yield estimates of SWAT. The model performs a search for the optimal or near-optimal parameter set using the sensitive parameters identified through the mechanisms described previously as decision variables. All other parameters are assigned nominal values based on information from the literature and prior knowledge of the watershed. Using the data collected for the watershed, the calibration model was executed for daily flow volume. Results for flow volume calibration are presented in Fig. 1. The search was conducted for an initial population of 150, 75 search generations, mutation rate of $20 \%$, and a binary tournament selection procedure. The values obtained for flow volume were then used during the search procedure for parameters that bring sediment yield closer to the measured data. Fig. 2 illustrates the calibration result for daily sediment yield, which was obtained using same GA parameters described for flow volume. The results reveal a relatively good match for flow volume with an $R^{2}$ value of 0.69 . The sediment fit seems reasonable as well with an $R^{2}$ value of 0.42 . Note, however, that no verification procedure was conducted due to lack of data. Additional data is currently being collected and will enable the authors to perform model verification in the future.

\section{Field-Scale Decision Support Models}

The computational models developed to operate at the field scale have the capability of identifying an optimal or near-optimal landscape, defined by land use types and farm management practices for all farm fields for; (1) single objective evaluation that minimizes erosion and sediment yield or maximizes net agricultural

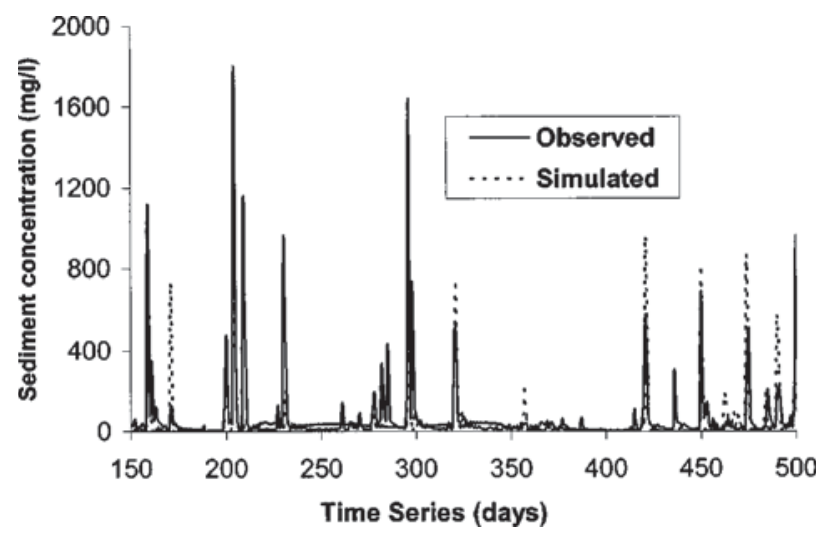

Fig. 2. Comparison of calibrated and measured values for sediment concentration

profit; and (2) multiobjective evaluation that minimizes erosion and sediment yield while simultaneously maximizing individual farm-based income that accrues from growing corresponding crops. While the approach used for these models is described here briefly, the reader is referred to Muleta and Nicklow (2002a) and Nicklow and Muleta (2001) for additional details.

\section{Linkage and Search Methodology}

Since both the GA and SPEA are search techniques that mimic the principle of evolution, the single objective and multiobjective models share many common features. The definition of genes, representation of chromosomes (i.e., alternative decision policies), evaluation of objective function(s) for the corresponding chromosomes, and the technique for linking and integrating the corresponding search algorithm with the SWAT model are similar for both the single objective and multiobjective models. Priorities considered during the integration of the simulation model and the search techniques were controlling computational time by using only simulation subroutines during the search, preserving originality of the simulation model so as to minimize upgrading efforts, and incorporating flexibility to handle other objective functions through a modular design.

A subbasin, or HRU, which is assumed to represent a single farm field, is the spatial scale at which the decision-support system conducts the search for preferred land use and management operations. Under this assumption, a landowner's decision concerning land uses and tillage types will have no influence on the decisions made by neighboring landowners. Expressed differently, the methodology allows each landowner within the watershed to make independent decisions, but contributes toward the overall goal of minimizing sediment yield to a receiving water body. This approach supports ILEPA's recognition that watershed planning and management begins with the responsibility of farmers and other landowners who have ownership rights within the watershed. Their land use choices directly affect both their personal income and their shared responsibility to maintain environmental quality. Effective decision making in such cases should thus recognize different stakeholder perspectives.

In order to accommodate the effect of crop rotation in evaluating landscapes, it is assumed that a farm management policy dictates the seasonal sequence of crops to be grown on an individual farm field for a 3 -year time horizon. Decision variables, or genes, are cropping and tillage practice combinations for a particular HRU, which are implemented over seasons of the 3-year 
decision horizon. It should be emphasized here that, the previous models (Nicklow and Muleta 2001; Muleta and Nicklow 2002a) allowed growth of up to two crops per year. For this application, from interviews conducted with local farm officers, the percentage of farm fields used during winter seasons in the demonstration watershed was found to be insignificant. As a result, growth of only one crop a year is allowed in the current model. Unlike the previous models for which five sequential genes defined a chromosome, a decision alternative is defined by a sequence of only three genes, each corresponding to a respective combination of crop type and farming practice from the first to the third year. An operational management database is developed for all crops believed to be grown in the watershed. This database dictates the type of land cover chosen for a particular season; tillage type used; planting and harvest dates for the crop, chemical (i.e., fertilizer and pesticide) application dates and dosages; end of year operations; calibrated value of $\mathrm{CN}$ to be used in estimating surface runoff taking into account soil type in the HRU and crop type selected for the year and its tillage type; potential heat units for a particular crop to reach maturity, which heavily influences crop yield; and other practices. In addition, an economic database that supplies information on production expenses, both variable costs and fixed costs, and the selling price of all crops included in the decision process is developed. This economic information, along with the crop yield estimate provided by SWAT, is used for estimation of net profit that may be targeted in either the single objective optimization or in the multiobjective model.

The search for a most-favored landscape solution begins with randomly generated chromosomes, each consisting of three genes. The water quality and hydrologic simulator is then used to provide subbasin response for each chromosome when the search algorithm requires its solution. This response establishes the basis for assigning a measure of fitness for each chromosome. The technique for using the objective function value as a measure of fitness is straightforward for the single-objective optimization (i.e., GA). However, for multiobjective optimization (i.e., SPEA), fitness must be evaluated differently.

\section{Multiobjective Optimization}

Many realistic problems involve simultaneous optimization of several incommensurable and often conflicting objectives. For example, in the current field-scale multiobjective watershed management problem, the objectives involve minimizing sediment yield while maximizing agricultural income. However, land covers that have significant erosion protection capability are generally noncash crops that generate little to no income, hence degrading the economic objective. This is a typical behavior of many multiobjective optimization problems (MOPS), which makes them significantly different from single-objective optimization problems.

In single-objective optimization, the final solution is usually unique and clearly defined. However, the typical goal in multiobjective optimization is finding tradeoffs between competing objectives. These tradeoff solutions are referred to as nondominated solutions or Pareto-optimal solutions. Various methods exist for multiobjective optimization. Recently, EAs have become established as an alternative to the traditional methods of simple aggregation (see Srinivas and Deb 1994; Zitzler and Thiele 1999). The advantage of EAs for solving MOPs include their capability of searching large decision spaces, thus raising the likelihood of locating a global Pareto-optimal solution, and their generation of multiple tradeoffs in a single optimization run, unlike aggregation methods that demand multiple search runs. In using EAs, the only significant difference between single-objective and multiobjective evaluation is the method of assigning a fitness value so that the performance measure accurately determines the value of an alternative solution relative to its counterparts. In single-objective optimization, the objective function value itself can be used as a measure of fitness. However, in multiobjective evaluations, it is necessary to design a means of converting the multidimensional objective function into a scalar fitness measure. Based on techniques of mapping multiple performance values to a single fitness value, there are a wide variety of EA-based methods for solving MOPs (Fonseca and Fleming 2000).

Motivated by the diversity of multiobjective optimization algorithms and the lack of comparative performance studies of the different approaches, Zitzler et al. (2000) provided a systematic comparison of six multiobjective EAs. Test functions having features that pose difficulties for EAs with regard to convergence to a Pareto-optimal front (Deb 1999) were considered in the study. These properties include convexity, nonconvexity, discrete Pareto fronts, multimodality, deception, and biased search spaces. As such, the writers were able to systematically compare the approaches based on the different kinds of difficulties and determine more exactly where certain techniques are more advantageous or have problems. The conclusions of their study included a clear hierarchy of algorithms with respect to the distance to the Paretooptimal front. The SPEA was ranked first and outperformed all other algorithms on five of the six test functions, and ranked second on the sixth-test function, which incorporated a deceptive feature. Based on the results of this comprehensive comparison study, a SPEA has been coded and integrated into the solution methodology for the multiobjective watershed management problem. For specific details regarding SPEA, the reader is referred to Zitzler and Thiele (1999).

\section{Watershed-Scale Decision Support Models}

Convinced by the fact that the tremendous negative impacts of erosion and sedimentation could be effectively controlled by properly managing activities within the watershed, the U.S. government has implemented a number of corrective watershed-scale programs, such as the Conservation Reserve Program (CRP) and the Total Maximum Daily Load (TMDL) program. The objective of the CRP is to encourage abandonment of farming on highly erodible fields, whereas the TMDL program focuses on reducing pollution within watercourses identified as having contaminant loads greater than established TMDL criteria. For water bodies whose quality is impaired due to agricultural NPS pollutants, a viable method for pollutant reduction and meeting TMDL limits is through the alteration of existing or currently planned agricultural land use patterns, such as enrolling a certain percentage of farm lands in the watershed into conservation programs such as CRP.

The watershed-scale analysis is designed to identify the best set of HRUs (farm fields) to be enrolled under conservation programs in order to achieve a maximum desirable condition from environmental and/or economic perspectives. Specifically, the objectives considered are: (1) identifying the best set of farm fields in the watershed to be covered with the most environmentally conscious land use and management operation sequences so as to achieve the maximum possible sediment yield reduction from the watershed; (2) to identify HRUs that are agriculturally most profitable; and (3) to identify the set of HRUs that may achieve a 
maximum reduction in sediment yield from the watershed, with the least sacrifice in agricultural profit (i.e., most cost-effective alternative). For all the three cases, the decision-support system relies on the linkage between SWAT and a GA. Since the solution methodology implemented is similar for all three, only the third scenario, case (3), will be described further. The advantage of the previously described flexibility that was introduced in the linkage process of the field-scale decision-support models has been realized during the watershed-scale model development. Additional modifications required to SWAT were very minimal, and methodological differences between the field-scale and watershed-scale searches were handled primarily within the optimization code, as another GA was developed for the watershed-scale model.

For the watershed-scale search, a decision alternative or chromosome is defined as a set of randomly selected HRUs or farm fields, which are regarded as genes. The number of genes in a chromosome depends on the user specified percentage of HRUs in the watershed that need to be enrolled under the conservation program. For example, if the desire is to bring $10 \%$ of the farm fields in the watershed into the program, then the number of genes will be fixed as $10 \%$ of the number of HRUs in the watershed. HRUs whose existing land use is classified as forest, urban development, or wetlands were preserved and were not considered as alternatives. The sequence of final land use and management operations, that were identified as optimal or near-optimal from the perspective of reducing sediment yield or maximizing net agricultural profit in the field-scale analysis, is used as an initial input for the watershed level analysis. Therefore, the HRUs chosen would be assumed to be covered by corresponding preferred land use and management operations in determining the environmental and economic implication of enrolling this set of HRUs under a conservation program. Existing land uses are preserved for all remaining HRUs. Similar to the field-scale analysis, a 3-year decision period is considered here as well.

The mathematical formulation for the third watershed-scale scenario [i.e., case (3)] can be expressed as

$$
\text { Maximize } Z=\left(\frac{Y_{2}-Y_{1}}{P_{2}-P_{1}}\right)
$$

subject to the transition constraints;

$$
\begin{gathered}
Y=f\left(H, C_{s}, X_{s}, M_{s}, t, s\right) \\
P=f\left(H, C_{s}, X_{s}, M_{s}, R, t, s\right)
\end{gathered}
$$

and crop management constraints (e.g, crop rotation, harvesting, and planting dates) expressed generally in functional form as

$$
g\left(C_{s}, X_{s}, M_{s}, t, s\right) \leqslant 0
$$

where $Z$ represents the function to be maximized; $Y=$ average annual sediment yield at the outlet of the watershed over the 3 -year decision period; $P=$ net average annual economic benefit over the watershed; subscripts 1 and 2 correspond to $Y$ and $P$ values that result by covering the alternative solutions by the most environmentally favored land use and management practices and options that generate the best net agricultural profits, respectively; $H=$ set of HRUs to be enrolled under the conservation program, $C_{s}$ and $M_{s}$ represent crops planted and management practices implemented during season $s$ of year $t ; X_{s}=$ generic term that represents all other hydrologic and hydraulic factors that may affect sediment yield and crop yield during season $s$ of year $t$; and $R$ $=$ average market price for crop $C$ over the 3 -year decision period.
Once a chromosome is generated, the final field-scale solutions (i.e., land use and management options) for the environmental objective are assigned to the HRUs and corresponding sediment yield at the outlet of the watershed $\left(Y_{1}\right)$ and total net profit from all fields in the watershed $\left(P_{1}\right)$ are evaluated. $Y_{2}$ and $P_{2}$ are evaluated by assuming coverage of the HRUs by the economically favored land uses and management combinations, thus enabling determination of the fitness value $(Z)$ that is used in subsequent GA operations. The final solution corresponds to the most costeffective set of HRUs to be enrolled for the conservation programs. Ideally, selected HRUs will be those which yield more sediment when used to grow agricultural crops, yield significantly less sediment when covered by environmentally friendly land covers, and those whose agricultural productivity is very low, even when used to grow cash crops.

\section{Application Results and Discussion}

For demonstration of the field-scale and the watershed-scale models, the Big Creek watershed, along with model parameters obtained by the associated automatic calibration effort, is used. The field-scale single-objective decision support model was applied first. For both environmental and economic objectives, an initial population size of 100 and a maximum of 50 search iterations were allowed. These variables were fixed based on previous operational experience with these models. Implementation of more intensive (i.e., larger population and greater generations) searches resulted in very minimal improvement in final results. As one might naturally expect, for all agriculturally dominated HRUs included in the search, continuous use of Fescue grass, a typical grass grown on lands enrolled under CRP in Southern Illinois, over the 3-year period was identified as the best option from the perspective of reducing sediment yield. From an economic perspective, a sequence of soybean with conservational tillage-corn with conservational tillage-soybean with conservation tillage was favored for the majority (i.e., 41 of 52) of agricultural fields.

During the search, using the environmental objective, land uses obtained for each of the 52 fields at every search generation were applied and sediment yield at the outlet of the watershed was estimated. For the final generation, presumed to be the optimal or near-optimal solution, the sediment yield estimate at Perk's road station, a gauging station managed by the ISWS, was found to be 5,733 metric tons/year. The observed average annual sediment yield at the site was 9,426 metric tons/year from 1999 2001. These figures indicate that implementation of the preferred land use and farm management policies would result in a $39 \%$ reduction of sediment yield at the station. While this analysis provides policymakers with valuable information for formulating decisions, it is important to note, however, that such a policy may not be fully economically viable.

For the field-scale multiobjective computational model, an initial population of 100 chromosomes, a maximum of 100 generations, a mutation rate of $20 \%$ and a maximum of 8 niches were allowed during the search. For one particular HRU, the Pareto front corresponding to the final generation and cropping sequences for the extreme end solutions (i.e., points A and B) in the front are given in Fig. 3. These results clearly demonstrate the capability of the model in generating tradeoff solutions among the objectives considered. Solutions on the bottom left of the curve are relatively good from a sediment reduction perspective, but generate only a fair agricultural profit. Those on the top right of the front are economically productive but generate more sediment 


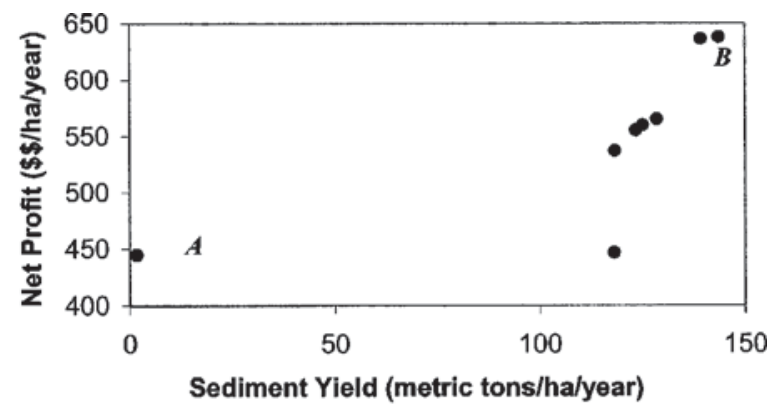

Solutions Corresponding to

Point A: HYNT HYNT HYNT (favors the environmental objective) Point B: SYST CRNT SYST (favors the economic objective)

where

HYNT: Hay with no tillage

SYST: Soybean with conventional spring tillage CRNT: Corn with no tillage

Fig. 3. Sample Pareto-optimal solution (final generation) for one hydraulic response unit

yield. The lack of alternatives in the middle of the curve is due to the extreme differences between field crops and perennial crops with respect to erosion protection and market prices and not an inadequacy of the SPEA in locating distributed nondominated solutions. It should also be noted here that actual economic figures may be slightly less than model results since no calibration is conducted for the crop yield estimate given by the model. Sufficient data for crop yield calibration simply do not exist.

For application of the watershed-scale model, the mostfavored land use and management combinations obtained during the single-objective field-scale searches were used as initial landscape. The objective function given in Eq. (1) is used for demonstration purposes, and an initial population of 250 chromosomes, a maximum of 50 search generations, and mutation rate of $20 \%$ were used for the application. It is assumed that $10 \%$ of farm fields can be entered into conservation programs, although any other percentage could be used depending on the application. A convergence plot of the application is given in Fig. 4, which indicates the progression of the search to a final solution. The optimal or near-optimal annual sediment yield at Perk's road station is found to be 7,636 metric tons. When compared to the observed sediment yield at the site, inclusion of $10 \%$ of the HRUs would result in a reduction of sediment yield by about $19 \%$. One could argue that this result, as well as the overall watershed-scale ap-

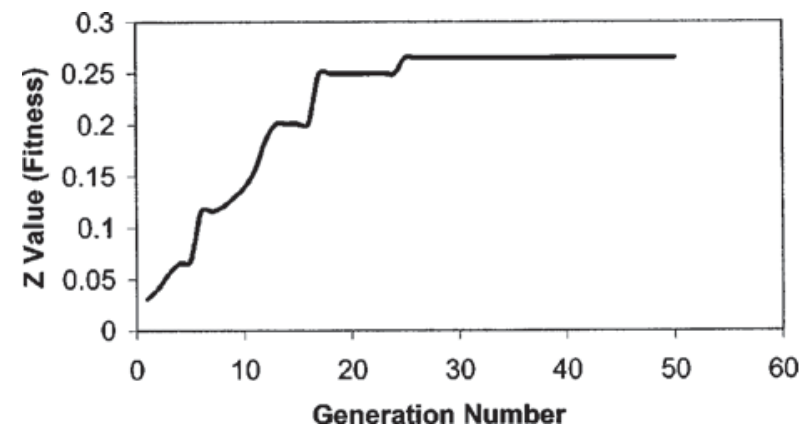

Fig. 4. Convergence plot for the watershed-scale search

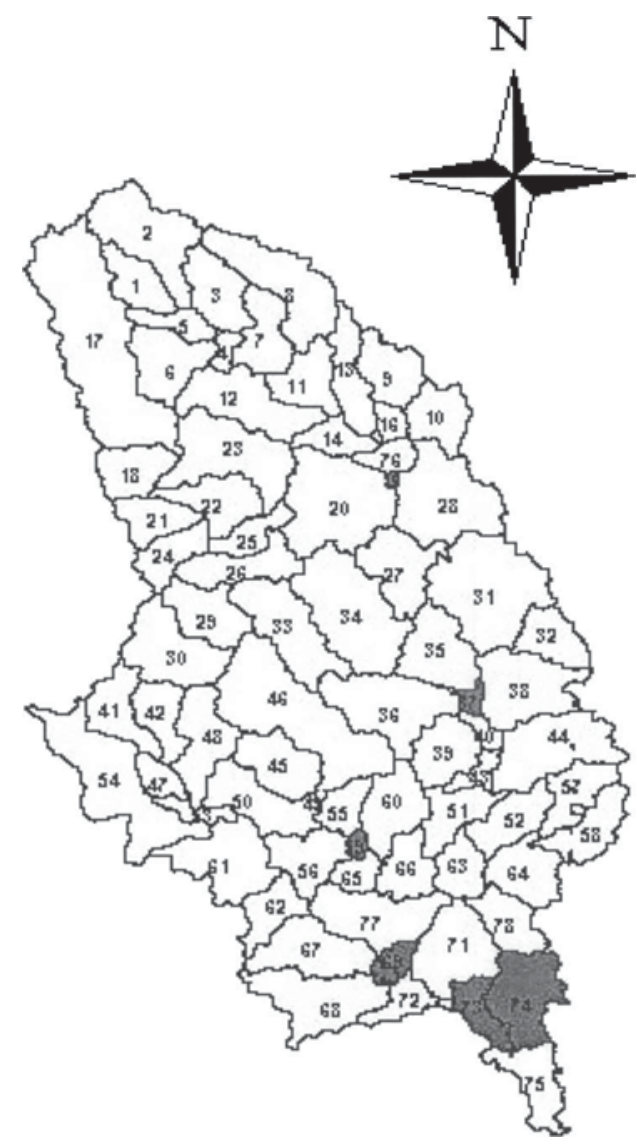

Fig. 5. Subbasins obtained for the search using Eq. (1)

proach, unfairly targets particular farms to reach a basin-wide objective. However, the total annual profit that may be generated from the watershed for the solution identified here was found to be $\$ 275,951$ and $\$ 253,459$ for the objectives that favor maximization of the net profit and minimization of sediment yield, respectively. The difference in the two figures is minimal, implying that inclusion of the chosen farm fields within conservation programs results in a limited loss of net profit while achieving a $19 \%$ reduction in sediment yield.

Fig. 5 provides the spatial distribution of the HRUs identified as optimal or near-optimal in the watershed scale analysis. Investigation of this distribution reveals that the most influential HRUs (i.e., those with larger area) identified are located closer to the outlet of the watershed. The HRUs chosen from the headwaters are of very small area and as such, their effect on $Z$ is relatively insignificant. This tendency is a direct consequence of the objective function $(Z)$ used in the analysis. The sediment yield $(Y)$ value used in Eq. (1) corresponds to the watershed outlet, and it may not be sensitive to activities carried out in HRUs located near the headwaters of the watershed. For watershed types termed "transport limited," FitzHugh and Mackay (2000) found that sediment yield at the outlet of the watershed mainly depends on the transport capacity of lower parts of channel networks and sediment yields from bottomland subbasins. At this stage of the research, no investigation of this phenomenon was carried out for Big Creek watershed. However, there is the possibility that the same reasoning has led to the spatial pattern given in Fig. 5. As a final analysis note, the search process was found to be extremely computationally intensive. For the GA parameters described, the watershed-scale search for example, required a central processing 
unit (CPU) time of 4.75 days on a $1.69 \mathrm{GHz}$, Pentium IV (PIV) personal computer (PC). On the same $\mathrm{PC}$, the multiobjective evaluation required approximately $53 \mathrm{~h}$. The computational demand is primarily due to the required iterative use of SWAT model in generating responses (i.e., objective function evaluation) to alternative landscapes. Concerned by the negative impact that the computational demand may impose on practical utility of the decision-support tools, an ANN-based model, with the capability to mimic required SWAT outputs, has been developed to serve as an auxiliary model during the search process.

\section{Artificial Neural Networks}

In the field-scale multiobjective decision-support model, the decision variables are land uses and corresponding farm management practices that need to be implemented in the farm fields of the watershed. This implies that all other environmental variables, such as climate conditions, soil type, watershed topography, and others that drive hydrologic processes, are constant during the search for preferred decision variables. Therefore, an approach that can model and provide reasonable estimates of required SWAT outputs (i.e., average annual sediment yield and net profit for the HRU) as a function of changing land use and management practices, with all other model variables and parameters kept fixed, and that can be executed faster than SWAT, could resolve concerns of excess computational time. Initiated by the growing popularity and effective application of neural networks for modeling nonlinear systems in various engineering and science disciplines, including water resources and hydrologic modeling, Muleta and Nicklow (2002c) investigated a multilayer feed-forward ANN for potential use as a replacement for SWAT in the fieldscale multiobjective decision-support model. Here, the ANN is applied based on the calibrated SWAT model and accounts for the management revisions previously described.

There are many types of ANNs, but all attempt to mimic the human brain. Analogous to humans, who learn from experience, knowledge in ANNs is gained through exposure to examples of the environment that they intend to model. This teaching mechanism, commonly known as training, is usually performed using the $\mathrm{BP}$ and the conjugate gradient methods, both of which are unfortunately local search algorithms and thus tend to become trapped at local optima. As with any gradient-based technique, the quality of their solutions depends on initial randomly drawn weights. In addition, the design of ANN architecture (i.e., number of layers, and number of nodes on a layer) in such approaches requires a trial-and-error procedure, which is a tedious, timeconsuming, and unreliable procedure. One way to overcome these drawbacks is the adoption of EAs in the training process. However, using EAs alone can be computationally intensive. Here, we describe a hybrid training technique that is formulated in such a way that EP determines the architecture and weights of the ANN, which correspond to region of global optima, after which BP is applied to fine tune the search in the overall region identified by EP. This EP-BP hybrid-training algorithm takes advantage of each algorithm's strength in overcoming weaknesses of the other. The effectiveness of the approach is demonstrated by the inspiring results presented herein. The trained ANN is then used as a replacement for SWAT in the watershed-scale decision-support tool, which results in a tremendous reduction in computational time needed for identifying most-favored watershed management solutions.

\section{Evolutionary Programming}

EP starts searching for optimal or near-optimal solutions by randomly generating feasible individuals within the given static or dynamic environment. Each of these initially chosen individuals undergoes a mutation process to generate offspring, one for each individual. The mutation approach is based on the conception that whatever genetic information transformations occur in EP, the resulting change in each behavioral trait follows a Guassian distribution with a zero mean and a standard deviation equal to unity (Fogel 1994). EP does not use a crossover operator, which makes its use for ANN training very appealing (Yao and Liu 1997). In $\mathrm{EP}$, mutation is the primary means of creating offspring. Fitness evaluation is then performed for both parent alternatives and the newly created individuals. The current population (i.e., original parents and newly generated individuals) are ranked in ascending order of their fitness values, for the minimization case. Then a selection operator is performed in such a way that individuals of higher fitness value would be given a higher probability of being selected. Individuals of the new generation will then be allowed to undergo the mutation step to create offspring. This cycle of creating individuals by mutation, ranking candidate solutions, and selection among the subset of offspring and parents continues until a stopping criterion is satisfied. For further details on EP, the reader is referred to Fogel (1999).

\section{Training Mechanism and Results}

To generate training data, a number of land use and management practices were randomly selected and assumed to have been exercised in the corresponding HRUs. The generated alternatives represent decision variables and are used as inputs for the ANN. The corresponding outputs (i.e., average annual sediment yield and average annual net profit) are estimated by SWAT, which in turn represent the desired outputs in the training process. 150 of these pairs were used as training data for determining connection weights and ANN architecture. Another 100 pairs were used as cross-training data and yet another 100 pairs as verification data. The inputs, as well as outputs, were standardized based on Haykin's (1999) recommendation. Output standardization was done in such a way that the values lie within the range of the activation function used in the training with some offset. The resulting inputs were standardized so that all inputs lie within a range of \pm 0.95 . Since the activation function used in training is the sigmoid function, which is bounded between 0 and 1 , the output data sets were standardized so that they lie within the range of 0.05 to 0.95 , allowing an offset of 0.05 from both extremes. The remainder of the training procedure is very similar to the method described by Muleta and Nicklow (2002c) in which the reader can obtain additional training details.

In this work, a population of 1,000 individuals, a maximum of 100 generations, a maximum of six hidden layers and a minimum of 1 hidden layer, a maximum of 15 nodes for each hidden layers and a minimum of 1 node, and a maximum and minimum weight of 2 and -2 , respectively, has been adopted for the EP algorithm. Using the weights and ANN architecture identified during the modest search of EP, the BP algorithm (Rumelhart et al. 1986) is subsequently applied as a secondary training step. Similar to EP, learning in BP results from the presentation of a prescribed set of training examples. Cross-training and validation data sets are also essential in application of BP training. Final weight vectors iden- 


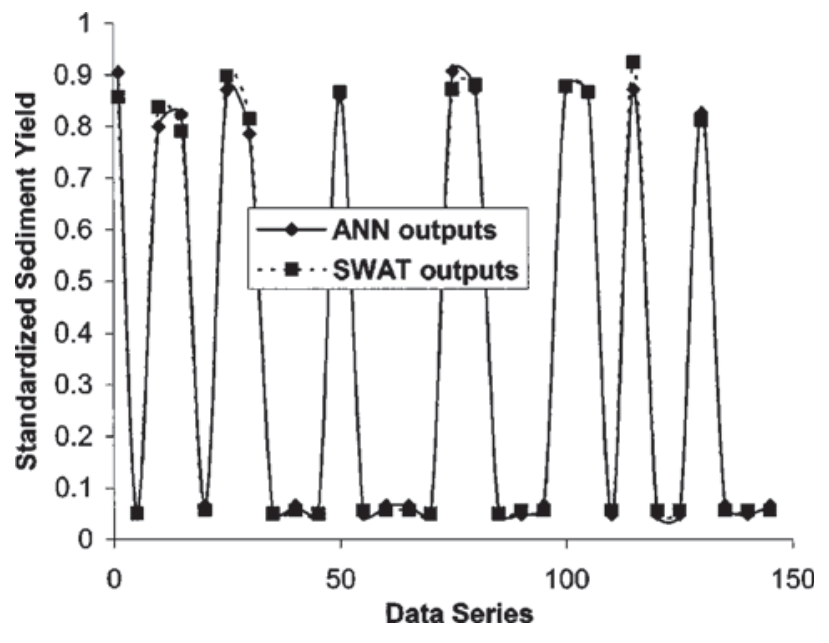

Fig. 6. Comparison of artificial neural network-simulated and soil and water assessment tool-simulated sediment yield for training data sets

tified by the BP algorithm for the ANN architecture determined by EP have subsequently been used in the watershed-scale decision support model.

Figs. 6 and 7 illustrate a comparison of the ANN-simulated and SWAT-estimated sediment outputs for the training data for sediment yield and net profit, respectively. The average value of Nash-Sutcliffe $R^{2}$ efficiency criteria (Nash and Sutcliffe 1970) was found to be 0.99 and 0.97 for training and verification of sediment yield for the 52 agriculturally dominated HRUs of the watershed. For net profit, the average Nash-Sutcliffe $R^{2}$ efficiency value all over the HRUs included in the search was found to be 0.95 and 0.86 for training and verification, respectively. The worst Nash-Sutcliffe $R^{2}$ value found was 0.98 and 0.83 for training and verification of sediment yield, and 0.85 and 0.68 for training and verification of net profit. Using a PIV, $1.69 \mathrm{GHz}$ PC, the training and data generation processes required a CPU time of $3.34 \mathrm{~h}$ and $5 \mathrm{~h}$, respectively. Impressed by the performance of the training algorithm and the capability of ANN in reproducing the output required during the search for preferred landscapes, SWAT was then replaced by the trained ANN. The search for solutions using the ANN model took only 4 min. Including data generation $(5 \mathrm{~h})$, training $(3.34 \mathrm{~h})$, and the search for final solutions (4 min),

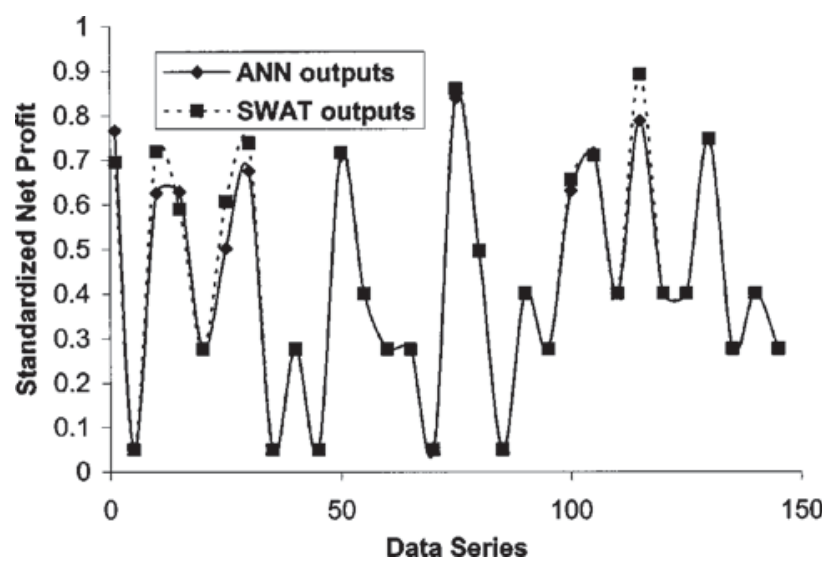

Fig. 7. Comparison of artificial neural network-simulated and soil and water assessment tool-simulated net profit for training data sets replacement of SWAT by the ANN model has resulted in an $84 \%$ reduction of CPU time for the field-scale multiobjective search process. The role of the ANN model may have an even greater impact when applied to the watershed-scale problem, which is computationally much more demanding. Future work will embark on extending the ANN model to the watershed scale search process.

\section{Conclusions}

A comprehensive decision-support system and methodology that has the capability to assist policymakers with watershed management decisions has been developed by integrating a well known watershed simulation model with EAs. SWAT has been integrated with both a GA and SPEA for single-objective and multiobjective problems, respectively. The overall model can be applied for watershed-scale, as well as field-scale, analysis. In addition, the watershed simulation model has been calibrated with an automatic calibration algorithm that is based on a GA.

Application of parameter reduction techniques, including parameterization and sensitivity analysis, have successfully screened the must-be-calibrated model parameters. The sensitivity analysis has been carried out using a stepwise regression method based on data generated with Latin hypercube sampling. Application of the decision-support system to the Big Creek watershed in Southern Illinois indicates their viability and their capability to address their corresponding objectives. The models were, however, found to be computationally demanding. Concerned by the impact of the CPU demand on the practicality of the computational tools, an ANN-based simulation model that mimics and generates required SWAT outputs was developed. The ANN model has been trained with a hybrid of EP and the BP algorithms. The training algorithm was found to be effective and efficient, and the replacement of SWAT by the trained ANN model resulted in an $84 \%$ reduction of CPU time.

The applications presented in this study clearly demonstrate the tremendous multifaceted role that EAs and artificial intelligence techniques could play in solving complex and realistic problems in environmental and water resources systems. GAs, EP, and SPEA, all of which are based on the principle of natural selection, have been used conjuctively for various purposes and applications. An ANN, a technique inspired by the working mechanisms of the human brain, has been successfully used to address the concern of computational demand. A novel training approach that exploits the strong features of both gradient-based and EA-based search approaches has been incorporated and could be used for applications to other systems. The computational models presented herein could also be extended to the management of other NPS pollutants, such as various species of nitrogen, phosphorus, and pesticides, thus making the models even more comprehensive. Future study will focus on model verification and investigation of model uncertainty due to various sources. Sensitivity of model outputs at various locations of the river network as a result of activities throughout the HRUs of the watershed will also be addressed in upcoming phases of the study.

\section{Acknowledgment}

The writers wish to thank the Illinois Council for Food and Agricultural Research (CFAR) for their support of this ongoing re- 
search effort, and the anonymous reviewers for their valuable input.

\section{References}

Arnold, J. G., Srinivasan, R., Muttah, R. S., and Williams, J. R. (1998). "Large-area hydrologic modeling and assessment. I: Model development." J. Am. Water Resour. Assoc., 34(1), 73-89.

Deb, K. (1999). "Multiobjective genetic algorithms: Problem difficulties and construction of test problems." Evol. Comput., 7(3), 205-230.

Demissie, M., Knapp, V. H., Parmer, P., and Kriesant, D. J. (2001). "Hydrology of the Big Creek Watershed and its influence on the Lower Cache River." Contract Rep. No. 2001-06, Illinois State Water Survey, Champaign, Ill.

FitzHugh, T. W., and Mackay, D. S. (2000). "Impacts of input parameter spatial aggregation in an agricultural nonpoint source pollution model." J. Hydrol., 236, 35-53.

Fogel, D. B. (1994). "An introduction to simulated evolutionary computation." IEEE Trans. Neural Netw., 5(1), 3-14.

Fogel, L. J. (1999). Intelligence through simulated evolution: Forty years of evolutionary programming, Wiley, New York.

Fonseca, C. M., and Fleming, P. J. (2000). "Multiobjective optimization." Evolutionary computation 2, advanced algorithms and operators. T. Back, D. B. Fogel, and Z. Michalewicz, eds., Institute of Physics, Philadelphia.

Goldberg, D. E. (1989). Genetic algorithms in search, optimization and machine learning, Addison-Wesley, Reading, Mass.

Guetersloh, M. (2001). Big Creek Watershed restoration plan, A component of Cache River Watershed resource plan, Illinois Dept. of Natural Resources, Springfield, Ill.

Haykin, S. (1999). Neural networks: A comprehensive foundation, Prentice-Hall, Upper Saddle River, N.J.

Helton, J. C., and Davis, F. J. (2000). "Sampling-based methods." Sensitivity analysis, A. Saltelli, K. Chan, and E. M. Scott, eds., Wiley, New York.

Holland, J. H. (1975). Adaptation in natural and artificial systems. University of Michigan Press, Ann Arbor, Mich.

Illinois Department of Natural Resources (IDNR). (1998). The pilot watershed program: Watershed management, monitoring, and assessment, Illinois Department of Natural Resources, Springfield, Ill.

Iman, R. L., and Conover, W. J. (1979). "The use of rank transform in regression." Technometrics, 21, 499-509.

Muleta, M. K., and Nicklow, J. W. (2002a). "Evolutionary algorithms for multiobjective evaluation of watershed management decisions." $J$. Hydroinformatics 4(2), 83-97.

Muleta, M. K., and Nicklow, J. W. (2002b). "Genetic algorithms for automatic calibration of physically-based distributed watershed mod- els." Proc., 2002 Conf. of the Environmental and Water Resources Institute, ASCE, Roanoke, Va.

Muleta, M. K., and Nicklow, J. W. (2002c). "Artificial neural networks for efficient decision making in watershed management systems." Proc., 2002 Conf. of the Environmental and Water Resources Institute, ASCE, Roanoke, Va.

Nash, J. E., and Sutcliffe, J. V. (1970). "River flow forecasting through conceptual models. I: A discussion of principles." J. Hydrol., 125, 277-291.

Nicklow, J. W., and Muleta, M. K. (2001). "Watershed management technique to control sediment yield in agriculturally dominated areas." Water Int., 26(3), 435-443.

Refsgaard, J. C. (1997). "Parameterization, calibration and validation of distributed hydrologic models." J. Hydrol., 198, 69-97.

Refsgaard, J. C., and Knudsen, J. (1996). "Operational validation and intercomparison of different types of hydrologic models." Water Resour. Res., 32(7), 2189-2202.

Rumelhart, D. E., Hinton, G. E., and Williams, R. J. (1986). "Learning internal representations by error propagation." Parallel distributed processing, Vol. 1, MIT Press, Cambridge, Mass.

Santhi, C., Arnold, J. G., Williams, J. R., Srinivasan, R., and Hauck, L. M. (2001). "Validation of the SWAT model on a large river basin with point and non point sources." J. Am. Water Resour. Assoc., 37(5), $1169-1188$.

Schwefel, H.-P. (2000). "Advantages (and disadvantages) of evolutionary computation over other approaches." Evolutionary computation 1, Basic algorithms and operators. T. Back, D. B. Fogel, and Z. Michalewicz, eds., Institute of Physics, Philadelphia.

Senarath, S., U. S., Ogden, F. L., Downer, C. W., and Sharif, H. O. (2000). "On the calibration and verification of two-dimensional, distributed, Hortonian, continuous watershed models." Water Resour. Res., 36(6), 1495-1510.

Srinivas, N., and Deb, K. (1994). "Multiobjective optimization using nondominated sorting in genetic algorithms." Evol. Comput., 1(2), 127149.

U.S. Environmental Protection Agency (USEPA). (2000). "Water quality conditions in the United States: A profile from the 1998 national water quality inventory report to congress." EPA-841-F-00-006, U.S. Environmental Protection Agency, Office of Water (4503F), Washington, D.C.

Yao, X., and Liu, Y. (1997). "Fast evolution strategies." Contr. Cybernet., 26(3), 467-496.

Zitzler, E., and Thiele, L. (1999). "Multiobjective evolutionary algorithms: A comparative case study and the strength Pareto approach." IEEE Trans. Evol. Comput., 3(4), 257-271.

Zitzler, E., Thiele, L., and Deb, K. (2000). "Comparison of multiobjective evolutionary algorithms: Empirical results." Evol. Comput., 8(2), 173-196. 